\title{
MODELOS DE NEGÓCIOS: ANÁLISE DA PRODUÇÃO CIENTÍFICA INTERNACIONAL NAS BASES DE DADOS WEB OF SCIENCE E SCOPUS NO PERÍODO DE 2010 A 2019
}

\author{
BUSINESS MODELS: ANALYSIS OF INTERNATIONAL SCIENTIFIC PRODUCTION IN THE \\ WEB OF SCIENCE AND SCOPUS DATABASES FROM 2010 TO 2019
}

\section{Luciana Aparecida Barbieri da Rosa $[10$}

Universidade Federal de Santa Maria, Santa Maria, RS, Brasil, lucianaaparecidabarbieri@yahoo.com.br

\author{
Maria Carolina Martins Rodrigues
}

Universidade de Algarve, Portugal, rodriguescarolina@live.com.pt

\section{Caroline Rossetto Camargo}

Universidade Federal de Santa Maria, Santa Maria, RS, Brasil, carolinerc@gmail.com

\section{Waleska Yone Yamakawa Zavatti Campos}

Pontifícia Universidade Católica do Rio de Janeiro, Rio de Janeiro, RJ, Brasil, waleskazavatti@alumni.usp.br

\author{
Larissa Cristina Barbieri
}

Faculdade São Luís, Sâo Paulo, SP, Brasil, barbierila@hotmail.com

\begin{abstract}
Resumo: O presente artigo apresenta os resultados de uma meta-análise que objetivou demonstrar como o campo de estudos internacional sobre "Modelos de Negócios" foi tratado por pesquisadores, por meio do apontamento das características das publicaçôes no período de 2010 - 2019, evidenciando a importância do conceito para a sociedade acadêmica atual. A pesquisa foi realizada nas bases de dados Web of Science e Scopus, com vistas à identificação das principais áreas temáticas, autores, tipos de documentos, título das fontes, países, anos de publicação, instituições e idiomas. Para isso, adotou-se na metodologia pesquisa bibliométrica, descritiva nas bases de dados Web of Science e Scopus, para seleçâo dos artigos que descrevem o tema deste estudo. Os resultados da pesquisa mostram que houve um crescimento do número de publicaçōes no período em estudo, um tema em franca expansão. Verificase que, o jornal Lecture Notes in Computer Science, apresenta o maior número de publicaçóes, e dos autores que mais publicaram sobre o assunto destacam-se Ghezzi, A. na Scopus e Parida, V. na WoS. As conclusóes mostram trajetórias produtivas para a pesquisa na área
\end{abstract}

Palavras-chave: Modelo de Negócios. Bibliometria. Web of Science, Scopus.

Abstract: This article presents the results of a meta-analysis that aims to demonstrate how the international field of studies on "Business Models" was treated by researchers, by pointing out characteristics of publications in the period from 2010 to 2019, highlighting the importance of the concept for the current academic society. A search was carried out in the Web of Science and Scopus databases, with a view to identifying the main thematic areas, authors, types of documents, title of sources, countries, years of publication, institutions and languages. For this, I adopted the bibliometric research methodology, descriptive in the Web of Science and Scopus databases, to select articles that describe the theme of this study. The results of the research show that there was an increase in the number of publications in the period under study, a topic in rapid expansion. It appears that the newspaper Lecture Notes in Computer Science, presents the largest number of publications and the authors that most publish 
on the subject in focus Ghezzi, A. in Scopus and Parida, V. in WoS. The conclusions show productive trajectories for research in the area.

Keywords: Business Models. Bibliometry. Web of Science. Scopus.

\section{Introdução}

As empresas realizam suas atividades em ambiente de elevada concorrência, de maneira que as mudanças nas economias industriais, informaçóes e conhecimento são essenciais para o crescimento do negócio e o melhoramento de produtos e serviços. A competência criada pelas organizaçóes constitui-se na otimização de todo seu ativo central, produtivo e estratégico, característica fundamental na era da informação (Orofino, 2011).

O conceito de modelo de negócios vem ganhando importância no ambiente empresarial como também nas instituiçóes de ensino superior. O termo surgiu na década de 1990, e começou a ser definido a partir do momento em que a tecnologia da informação, em especial, a internet passou a influenciar os negócios (Osterwalder e Pigneur, 2009).

Osterwalder e Pigneur (2011) definem modelo de negócios como a descrição clara das atividades de oferta de produtos e serviços de uma empresa, auxiliando na forma de realizar negócios sob condiçôes de incerteza. $\mathrm{O}$ modelo de negócio apresenta quem são os clientes e quais as estratégias que a empresa pode utilizar para se manter competitiva a partir da criação de valor aos seus clientes (Abraham, 2013).

Uma das ferramentas mais utilizadas nesse contexto é o "Business Model Canvas (BMC)" que segundo Dorf e Blank, (2012, p. 571):
... é uma importante ferramenta de gerenciamento estratégico para empreendimentos. O BMC ajuda no desenvolvimento de esboços e versóes finais de modelos de negócio, tanto para um novo empreendimento quanto para uma empresa que já está na ativa. Trata-se de um mapa visual que otimiza a visualização e compreensão do modelo de negócio de um empreendimento, dividindo-o em nove importantes blocos para o detalhamento de um negócio.

Deste modo, considerando a importância dos modelos de negócios para as empresas, e o impacto no conhecimento científico no que tange em questóes práticas e gerenciais, coloca-se a seguinte questáo de pesquisa: Como se apresenta o cenário das publicaçóes internacionais sobre modelos de negócios nas bases de dados Web of Science e Scopus?

Partindo da premissa de investigação, este artigo tem como objetivo apresentar o cenário das publicaçóes internacionais sobre modelos de negócios nas bases de dados Web of Science e Scopus no período compreendido entre 2010 e 2019, tendo como objetivos específicos: (1) analisar as características das publicaçóes que se relacionam ao tema Modelos de Negócios; (2) apresentar a caracterização da produção científica e dos aspectos metodológicos de investigação.

$\mathrm{O}$ artigo estrutura-se, além da introdução, em cinco seçóes. $\mathrm{Na}$ segunda seção são apresentados os aspectos que descrevem os modelos de negócios. Na terceira seção são descritos os procedimentos metodológicos adotados na realizaçáo do presente trabalho. Na quarta seçáo são apresentados os principais resultados. $\mathrm{Na}$ quinta seção são expostas as consideraçôes finais, e por fim, as limitaçôes e futuras linhas de investigação encerram a sexta seção. Por último temos a lista das referências utilizadas neste trabalho. 


\section{Perspectivas teóricas sobre distintos conceitos de modelo de negócios e suas taxinomias}

Com a expansão do uso da internet e das tecnologias de informação ocorreu um número crescente de representaçôes e possibilidades de negócios. Com isso, novas configuraçôes organizacionais foram criadas, permitindo que empresas se beneficiem da criação conjunta de valor e de distintos canais e redes de distribuição (Osterwalder, 2004).

De acordo com Campos (2010) o termo "modelo de negócio" começou a ser discutido na literatura em 1957, quando surgiu o primeiro artigo sobre o assunto. Foi Peter Drucker (1973), no entanto, quem escreveu o trabalho "Teoria do Negócio" e que seria considerado um marco sobre o assunto. No texto, o autor discute a teoria do negócio e inicia um debate teórico sobre os pressupostos sobre os quais as empresas são construídas.

Somente alguns anos depois, na década de 1990, o conceito de modelo de negócio passou a ser amplamente difundido e utilizado. Com a ascensão comercial da internet, muitos novos empreendedores estavam surgindo, situação na qual os empresários das chamadas "empresas ponto com" tentavam encontrar seu lugar no mundo dos negócios com o objetivo de influenciar investidores a financiá-los. A síntese de ideias onde se fundamentava a lógica de funcionamento dos empreendimentos, dos empreendedores e dos investidores era chamada de modelo de negócio (MAGRETTA, 2002).

Os modelos de negócio devem definir e articular a descrição de objetos estratégicos que melhoram as discussôes relacionadas à tomada de decisão, possibilitando que se tornem cada vez mais complexas (Osterwalder e Pigneur, 2013). Hamel (2000) sugere que o modelo de negócio deveria descrever as forças distintas de uma organização ao fazer negócio, enquanto que para Lind (2004) é a capacidade de comunicar de maneira concisa as forças do negócio, o que diferencia modelo de negócio de plano de negócio.

Para Chesbrough e Rosenbloom (2002) o modelo de negócio estabelece a maneira pela qual a organização conseguirá ser competitiva no setor de atuação. Segundo os autores, o modelo de negócio deve seguir alguns passos: 1) articular a proposição de valor aos usuários; 2) identificar os mercados-alvo; 3) determinar as formas de obtenção de receitas; 4) definir a estrutura da cadeia de valor necessária à criação de distribuição do valor; 5) descrever os recursos necessários para a criação da cadeia de valor; 6) demonstrar a estrutura de custos e o lucro potencial; 7) posicionar a empresa no mercado, conectando a empresa aos fornecedores e clientes; e 8) orientar a organização na formulação de estratégias competitivas para obter vantagens frente aos concorrentes.

O modelo de negócio permite que uma nova tecnologia seja transformada em valor econômico, pois agrupa atividades e componentes essenciais para criação de valor aos consumidores, ao mesmo tempo em que permite a apropriação de parte desse valor. Conhecer os componentes do modelo de negócio de uma organização facilita a percepção de mudanças que precisam ser realizadas (Osterwalder, 2004).

O modelo de negócio é composto por uma variedade de elementos estratégicos como produtos, clientes, tecnologia, mercado e recursos (Baden-Fuller; Morgan, 2010). Os autores também afirmam que um modelo de negócio não é feito somente pelos componentes, mas pela forma como são organizados e integrados dentro de uma empresa, objetivando atingir um determinado resultado. 
Neste contexto Lambert (2006, p.2) argui que "os modelos de negócio são conceitos abstratos e complexos cuja compreensão pode ser reforçada através do desenvolvimento de um esquema de classificação geral", e para tanto, são utilizadas taxonomias, que podem ser definidas como classificaçóes criadas a partir da realidade empírica. Ainda de acordo com o autor, a classificação envolve a ordenação de objetos em classes ou grupos baseados em sua similaridade e no reconhecimento de diferenças e semelhanças. A tipologia é uma classificação inerente à pesquisa dedutiva, já a taxonomia é relativa à pesquisa indutiva com o objetivo de criar grupos homogêneos. Existem diferentes visóes e componentes de modelos de negócio.

Nesse cenário, o modelo de negócio proposto por Osterwalder (2004) consiste numa representação importante de noves grupos. São eles: configuração de valor; capacitaçôes; parcerias; estrutura de custos; proposta de valor; fluxo de receitas; canais de distribuição; relacionamento; consumidor alvo.

Neste contexto, Zott e Amit (2010) desenvolveram um estudo teórico que investiga os fatores analisando a temática e relacionando com inovação e verificaram que a diferenciação e a liderança em custos auxiliam no desempenho empresarial. Osterwalder e Pigneur (2009) transformaram o esquema conceitual Business Model Canvas em um mapa visual, como uma ferramenta dinâmica para criaçáo, modificação, compreensão e inovação de modelos de negócios.

Já Teece (2010) desenvolveu um modelo de negócio voltado para a inovação. Nesse caso, sobrevém a definição de inovação por meio de modelos de negócios, ou "modelos de negócios inovadores". Ainda neste contexto, Schneider e Spieth (2013), na sua pesquisa sobre modelo de negócios inovadores, alegam que essa definição surgiu nas pesquisas no momento em que os modelos de negócios não podiam ser considerados como modelos estáticos, já que o cenário era marcado pela procura de inventividade constante como feedback ao ambiente concorrencial.

A vista disso, Boons, Lüdeke-Freund (2013) introduziu a concepção de sustentabilidade, argumentando que os modelos de negócio apresentam papel primordial no entendimento e no avanço da inovação sustentável das atividades-chave, sistemas de consumo e produção. Neste cenário Edgeman e Eskildsen (2014) destacam que as organizaçôes que vislumbram as premissas da sustentabilidade a longo prazo possuem como fundamental objetivo a qualidade superior a em seus produtos e serviços.

Além disso, Yang, et al (2017) complementam que a sustentabilidade é fator fundamental para viabilidade dos negócios no futuro e destacam a importância do valor não capturado para a inovação sustentável auxiliando nos ensejos voltados para os modelos de negócios sustentáveis.

Destarte, Evans, et al (2017) destacam a importância dos modelos de negócios sustentáveis e sua relação com a teoria de redes, teoria dos stakeholders e processos produtivos que integram o cerne da sustentabilidade cedidos e capturados na rede de valor. Nas palavras de Linder e Williander (2017), os modelos de negócios impactam em instigaçóes aos empreendedores, em virtude da remodelação de suas estratégias empresariais.

Centobelli, et al (2020) estuda o assunto voltado para a economia circular e enfatiza que é necessário para essa perspetiva o entrelaçamento dos stakeholders da cadeia de suprimentos e as propostas de valor aos clientes. 
Com isso, á luz dos estudos, algumas pesquisas discorrem sobre os fatores inibidores aos modelos de negócio sustentáveis, como, por exemplo, no estudo de Schaltegger, Hansen e Lüdeke-Freund (2016), que relataram a falta de envolvimento dos stakeholders no processo de proposição e criação de valor, e no de Boons, Lüdeke-Freund (2013) em relação à resistência na inserção de tecnologias em novos modelos de negócio.

Por fim, o conceito de modelos de negócios pode auxiliar as empresas a compreender, comunicar, compartilhar, alterar, medir, simular e aprender mais sobre os diferentes aspectos essenciais para a criação de valor empresarial. No próximo item será abordado o método do estudo.

\section{Metodologia}

Este estudo bibliométrico caracteriza-se como descritivo com abordagem quantitativa (Machado, 2007) no qual se utilizou como material de estudo os artigos científicos publicados entre os anos 2010 e 2019 sobre o tema Modelos de Negócio (Business Models).

\subsection{Definição do escopo do estudo}

Os dados foram recolhidos diretamente da base de dados das coleçóes Web of Science (WOS) do Institute for Scientific Information (ISI) e Scopus, duas das maiores bases multidisciplinares que englobam periódicos revisados por pares reconhecidos pela comunidade científica internacional. No campo de pesquisa foram inseridas as palavras "Business Models", assim, foram identificadas as características gerais das publicaçóes, com o objetivo de apresentar o contexto em que o tema é referenciado. Estas informaçóes foram tratadas em tabelas e gráficos de Excel para a sua análise.

Estas coleçôes de base de dados detêm jornais indexados, sendo, alguns dos mais citados nas suas respetivas áreas, dentre os quais destacam-se: Lecture Notes in Computer Science, Journal of Cleaner Production, Sustainability, Lecture Notes in Business Information Processing, Procedia Cirp, na Scopus e Journal of Cleaner Production, Sustainability, Procedia Cirp, Lecture Notes in Business Information Processing na WoS. Consideraram-se todos os artigos publicados no período de 2010 a 2019 , referidos na Quadro 1, resultando em 14.386 artigos, 10.283 proceedings paper (trabalhos previamente apresentados em conferências) e 1248 review (revistas).

Quadro 1 - Tipo de documentos

\begin{tabular}{|l|r|l|r|}
\hline Documentos & Scopus & WoS & \multicolumn{1}{|l|}{ Total } \\
\hline Abstract Report & 1 & 14 & 15 \\
\hline Article & 9973 & 4413 & 14386 \\
\hline Book & 440 & & 440 \\
\hline Book Chapter & 1741 & 28 & 1769 \\
\hline Book Review & & 11 & 11 \\
\hline Business Article & 3 & & 3 \\
\hline Conference Paper & 7393 & 2890 & 10283 \\
\hline Conference Review & 328 & & 328 \\
\hline
\end{tabular}




\begin{tabular}{|l|r|r|r|}
\hline Documentos & Scopus & WoS & \multicolumn{1}{|c|}{ Total } \\
\hline Data Paper & 1 & & 1 \\
\hline Early Access & & 31 & 31 \\
\hline Editorial & 107 & 141 & 248 \\
\hline Erratum & 15 & 3 & 18 \\
\hline Letter & 7 & 3 & 10 \\
\hline News Item & & 8 & 8 \\
\hline Others & 167 & & 167 \\
\hline Retracted & 8 & 1 & 9 \\
\hline Review & 887 & 361 & 1248 \\
\hline Short Survey & 117 & & 117 \\
\hline Total & $\mathbf{2 1 1 8 8}$ & $\mathbf{7 9 0 4}$ & $\mathbf{2 9 0 9 2}$ \\
\hline
\end{tabular}

Fonte: Elaboração própria.

Segundo Araújo (2006). A bibliometria é uma técnica de cunho quantitativo criada com o objetivo de avaliar a produtividade dos autores e efetuar estudos de citaçóes. Para Bufrem e Prates (2005) a bibliometria busca um perfil dos registos do conhecimento, utilizando um método quantificável para gerar informaçóes que mapeiam o desenvolvimento da produção científica. Já os autores Rousseau (2001) e Camps et al. (2006) definem bibliometria como uma disciplina que facilita o estudo quantitativo da produção científica, através da análise da sua própria natureza e da transferência de uma ciência em determinado período.

De acordo com Rousseau e Rousseau (2000) o estudo bibliométrico ou bibliometria tem por base três leis: Lei de Lotka ou Lei do Quadrado Inverso (mede a produtividade de autores), Lei de Bradford ou Lei da Dispersão (mede a produtividade de jornais) e Lei de Zipf ou Lei do Mínimo Esforço (consiste em medir a frequência de ocorrência de palavras)

$\mathrm{Na}$ análise bibliométrica do presente estudo procurou-se identificar as variáveis seguintes: principais autores, título das fontes, principais instituiçóes, ano das publicações, principais países e idiomas, áreas do conhecimento e a relação entre autores com mais publicaçôes e as obras mais citadas.

\subsection{Etapas da recolha dos dados}

Este estudo dividiu-se em duas etapas: foram inseridos os termos "Business Models", no campo de pesquisa das bases WOS e Scopus, delimitando-se o período entre 2010 e 2019. Procedeu-se posteriormente ao levantamento das características gerais das publicaçóes.

$\mathrm{Na}$ segunda etapa, as publicaçóes mais citadas foram comparadas com os autores que mais publicaram no mesmo período. A Figura 1 evidencia as etapas da pesquisa. 
Figura 1 - Etapas da pesquisa

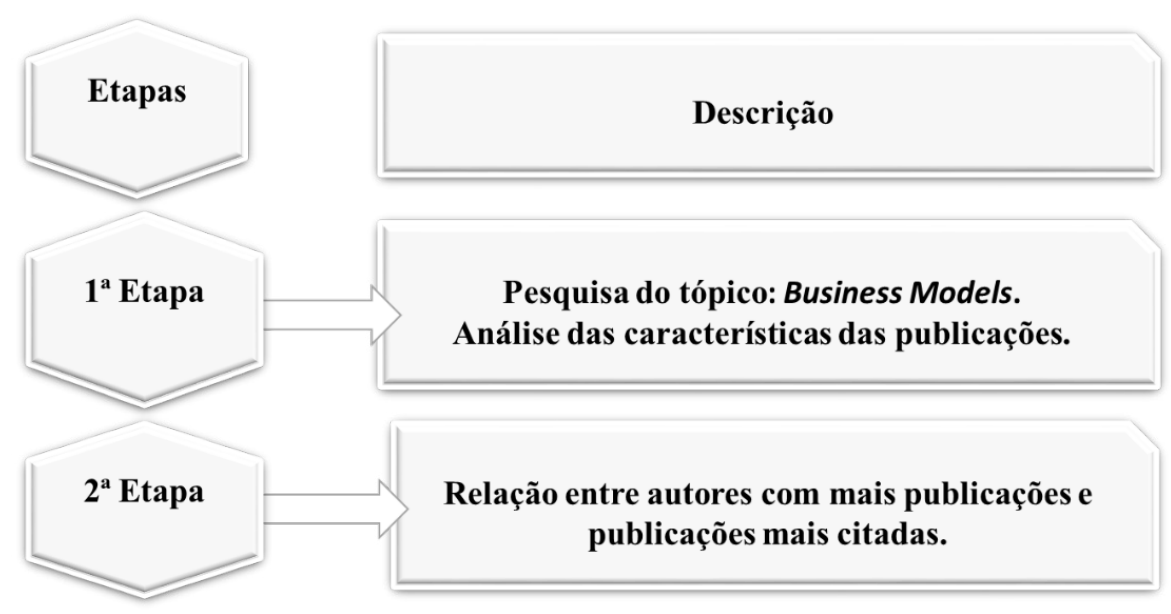

Fonte: Elaboração própria.

A análise bibliométrica do presente estudo foi realizada de acordo com as etapas descritas na Figura 1. No próximo item serão apresentados os resultados do estudo.

\section{Resultados}

Os resultados do estudo a seguir apresentados identificam as principais características da produção científica na base de dados das coleçóes WOS e Scopus relacionados com a palavra-chave "Business Models" no período 2010 a 2019. Depois de refinar a pesquisa relativamente às categorias de Business Models e ainda, optar por apresentar resultados referentes a artigos, obteve-se o total de 14.386 artigos. Serão apresentadas as características gerais das publicaçóes: principais autores, título das fontes, principais instituiçóes, ano das publicaçóes, principais países e idiomas e áreas de pesquisa. Depois, serão apresentados o número de publicações por autor e o número de citaçóes.

\subsection{Características gerais das publicaçôes}

Apresentam-se a seguir, as características gerais das publicaçóes relacionadas com os temas Business Models nas categorias de principais autores, título das fontes, instituiçôes, ano das publicaçôes, países, idiomas e áreas de pesquisa.

\subsubsection{Principais autores}

O Quadro 2 apresenta os dez principais autores que publicaram artigos com os temas Business Models no período analisado, em cada base de dados. 
Quadro 2 - Principais Autores

\begin{tabular}{|l|r|r|r|}
\hline \multicolumn{1}{|c|}{ Autores } & \multicolumn{1}{c|}{ Scopus } & \multicolumn{1}{c|}{ WoS } & \multicolumn{2}{c|}{ Total } \\
\hline Ahokangas, P. & 24 & 14 & 38 \\
\hline Bocken, N.M.P. & 26 & & 26 \\
\hline Bouwman, H. & 35 & & 35 \\
\hline Evans, S. & 32 & 16 & 48 \\
\hline Gassmann, O. & 21 & 13 & 34 \\
\hline Ghezzi, A. & 37 & & 37 \\
\hline Lindgren, P. & 35 & 16 & 51 \\
\hline Ludeke-Freund, F. & & 13 & 13 \\
\hline Parida, V. & & 23 & 23 \\
\hline Vale, Z. & 22 & 21 & 48 \\
\hline Yamakami, T. & & & 22 \\
\hline Yrjola, S. & 22 & & 14 \\
\hline Zarnekow, R. & & 15 & 22 \\
\hline Zhang, J. & & 14 & 15 \\
\hline Zhang, Q. & $\mathbf{2 8 1}$ & $\mathbf{1 5 9}$ & 14 \\
\hline Total & & & $\mathbf{4 4 0}$ \\
\hline
\end{tabular}

Fonte: Elaboraçáo própria.

Constata-se a existência de uma multiplicidade e diversidade quanto à autoria dos trabalhos envolvendo o tema analisado. Destacam-se os autores com mais artigos publicados: Lindgren, P., Evans, S., Vale, Z. com as 51, 48 e 48 publicaçóes respetivamente Desta forma, verifica-se a existência de vários investigadores que se destacam quando se analisa o tema de Business Models.

\subsubsection{Título das fontes}

O Quadro 3 apresenta as principais fontes e número de artigos publicados relacionados ao tema Business Models.

Quadro 3 - Quantidade de artigos por fonte

\begin{tabular}{|l|r|r|r|}
\hline Fonte & \multicolumn{1}{l|}{ Scopus } & WoS & \multicolumn{1}{l|}{ Total } \\
\hline Advances In Intelligent Systems And Computing & 98 & & 98 \\
\hline Communications In Computer and Information Science & 125 & & 125 \\
\hline Emerald Emerging Markets Case Studies & 107 & & 107 \\
\hline Energy Policy & 96 & 85 & 181 \\
\hline IFIP Advances Information Communication Technology & 154 & 64 & 218 \\
\hline Journal of Cleaner Production & 253 & 235 & 488 \\
\hline Lecture Notes In Business Information Processing & 232 & 100 & 332 \\
\hline Lecture Notes in Computer Science & 299 & 86 & 385 \\
\hline Long Range Planning & & 49 & 49 \\
\hline Procedia CIRP & 226 & 134 & 360 \\
\hline
\end{tabular}




\begin{tabular}{|l|r|r|r|}
\hline Renewable Sustainable Energy Reviews & 51 & 51 & 102 \\
\hline 1102 Sustainability & 236 & 186 & 422 \\
\hline Technological Forecasting And Social Change & 64 & 64 & 128 \\
\hline Total & $\mathbf{1 8 2 6}$ & $\mathbf{1 0 5 4}$ & $\mathbf{2 8 8 0}$ \\
\hline
\end{tabular}

Fonte: Elaboração propria.

Os jornais que mais publicaram envolvendo o tema, nas duas bases de dados, foram: Journal of Cleaner Production, Sustainability, Lecture Notes in Computer Science, Procedia CIRP, Lecture Notes in Business Information Processing. Esses indicios demosntram a importância do tema, visto que são discutidos em journals de auto impacto como pode ser visualizado por exemplo nos journals (Journal of Cleaner Production $e$ Sustainability). Demonstrando aos pesquisadores dessa temática onde se localizam os principais estudos

\subsubsection{Principais Instituiçóes}

Estão apresentadas no Quadro 4 as dez instituições que mais se destacaram e publicaram trabalhos relacionados com o tema.

Quadro 4 - Principais Instituições

\begin{tabular}{|c|c|c|c|}
\hline Instituiçóes & Scopus & WoS & Total \\
\hline Aalto University & 143 & 81 & 224 \\
\hline Aarhus Universitet & 100 & & 100 \\
\hline Bucharest University of Economic Studies & & 89 & 89 \\
\hline Delft University of Technology & 171 & 78 & 249 \\
\hline Lund University & & 53 & 53 \\
\hline Politecnico di Milano & 157 & & 157 \\
\hline Polytechnic University Of Milan & & 73 & 73 \\
\hline Royal Institute of Technology KTH & 113 & 67 & 180 \\
\hline School of Economics and Management & 99 & & 99 \\
\hline Technical University of Berlin & 101 & & 101 \\
\hline Tsinghua University & 101 & & 101 \\
\hline University of St Gallen & 130 & 6 & 186 \\
\hline University of California System & & 94 & 94 \\
\hline University of Cambridge & 154 & 89 & 243 \\
\hline University of London & & 94 & 94 \\
\hline Total & 1269 & 774 & 2043 \\
\hline
\end{tabular}

Fonte: Elaboração propria.

Dentre as dez instituições que mais publicaram, destacam-se: Aalto University (Espoo - Finlândia), University of Cambridge (Cambridge - Reino Unido), Delft University of Technology (Delft, Países Baixos), Universitat St. Gallen (St. Gallen, Suíça). Com isso, por meio dos dados apresentados acima evidenciam a abrangência e importância do tema em instituiçóes de ensino ao redor do mundo, bem como a representatividade das instituiçôes no contexto da temática sinalizando aos pesquisadores as principais instituiçóes que podem pesquisar. 


\subsubsection{Anos de Publicaçáo}

O Quadro 5 mostra o número de artigos das bases de dados relacionados com o tema Business Models, que foram publicados nos anos em estudo. Com base na figura, é nítido que é tema emergente e que vem crescendo anualmente, na medida em que as organizaçóes para se manterem competitivas necessitam remodelar seus modelos de negócios.

Quadro 5 - Anos de publicação

\begin{tabular}{|l|r|r|r|r|r|r|r|r|r|r|r|}
\hline Ano & $\mathbf{2 0 1 0}$ & $\mathbf{2 0 1 1}$ & $\mathbf{2 0 1 2}$ & $\mathbf{2 0 1 3}$ & $\mathbf{2 0 1 4}$ & $\mathbf{2 0 1 5}$ & $\mathbf{2 0 1 6}$ & $\mathbf{2 0 1 7}$ & $\mathbf{2 0 1 8}$ & $\mathbf{2 0 1 9}$ & Total \\
\hline Scopus & 1469 & 1513 & 1584 & 1799 & 1856 & 1987 & 2306 & 2597 & 2861 & 3216 & 21188 \\
\hline WoS & 322 & 355 & 360 & 489 & 518 & 839 & 1008 & 1185 & 1305 & 1358 & 7739 \\
\hline Total & $\mathbf{1 7 9 1}$ & $\mathbf{1 8 6 8}$ & $\mathbf{1 9 4 4}$ & $\mathbf{2 2 8 8}$ & $\mathbf{2 3 7 4}$ & $\mathbf{2 8 2 6}$ & $\mathbf{3 3 1 4}$ & $\mathbf{3 7 8 2}$ & $\mathbf{4 1 6 6}$ & $\mathbf{4 5 7 4}$ & $\mathbf{2 8 9 2 7}$ \\
\hline
\end{tabular}

Fonte: Elaboração própria.

\subsubsection{Principais países}

No Quadro 6 é apresentada a quantidade de artigos distribuídos pelos principais países.

Quadro 6 - Quantidade de artigos por país

\begin{tabular}{|l|r|r|r|}
\hline \multicolumn{1}{|c|}{ Países } & \multicolumn{1}{c|}{ Scopus } & \multicolumn{1}{c|}{ WoS } & \multicolumn{1}{c|}{ Total } \\
\hline Australia & 717 & 293 & 1010 \\
\hline China & 1727 & 527 & 2254 \\
\hline Finland & & 366 & 366 \\
\hline France & 813 & & 813 \\
\hline Germany & 2315 & 961 & 3276 \\
\hline India & 756 & & 756 \\
\hline Italy & 1161 & 518 & 1679 \\
\hline Netherlands & 827 & 376 & 1203 \\
\hline Spain & 953 & 453 & 1406 \\
\hline Sweden & & 380 & 380 \\
\hline United Kingdom & 2114 & 873 & 2987 \\
\hline United States & 3613 & 1180 & 4793 \\
\hline Total & $\mathbf{1 4 9 9 6}$ & $\mathbf{5 9 2 7}$ & $\mathbf{2 0 9 2 3}$ \\
\hline
\end{tabular}

Fonte: Elaboração própria.

Verificou-se que os países que mais artigos publicaram sobre modelos de negócios no período analisado foram: Estados Unidos, Alemanha, Reino Unido e China. Destaca, a partir desses dados os investimentos em pesquisas são em países desenvolvidos, não destacando nenhum pais em desenvolvimento. 


\subsubsection{Principais idiomas}

No que se refere aos idiomas dos trabalhos publicados na área de estudo, 27801 artigos estão publicados em inglês, conforme se demonstra no Quadro 7, correspondendo a 95,94\% dos trabalhos.

Quadro 7 - Quantidade de artigos por Idioma

\begin{tabular}{|l|r|r|r|}
\hline Idioma & Scopus & WoS & Total \\
\hline Chinese & 174 & 13 & 187 \\
\hline Croatian & & 7 & 7 \\
\hline Dutch & & 3 & 3 \\
\hline English & 20371 & 7430 & 27801 \\
\hline French & 86 & 6 & 92 \\
\hline German & 15 & 54 & 254 \\
\hline Italian & 21 & & 15 \\
\hline Japanese & 13 & & 21 \\
\hline Korean & & & 13 \\
\hline Polish & 79 & 7 & 7 \\
\hline Portuguese & 76 & 48 & 127 \\
\hline Russian & 221 & 37 & 113 \\
\hline Spanish & $\mathbf{2 1 2 5 6}$ & 116 & 337 \\
\hline Total & & $\mathbf{7 7 2 1}$ & $\mathbf{2 8 9 7 7}$ \\
\hline & & & \\
\hline
\end{tabular}

Fonte: Elaboração própria

\subsection{7 Áreas de pesquisa}

As áreas de pesquisa com maior número de publicaçôes foram ciência da computação, engenharia, negócios gestão e contabilidade, conforme Quadro 8.

Quadro 8-Áreas de pesquisa

\begin{tabular}{|c|c|c|c|}
\hline Áreas & Scopus & WoS & Total \\
\hline Business Economics & & 2644 & 2644 \\
\hline Business, Management and Accounting & 7430 & & 7430 \\
\hline Computer Science & 7126 & 1534 & 8660 \\
\hline Decision Sciences & 2017 & & 2017 \\
\hline Economics, Econometrics and Finance & 2935 & & 2935 \\
\hline Energy & 1884 & 446 & 2330 \\
\hline Engineering & 5777 & 1873 & 7650 \\
\hline Environmental Science & 1695 & 839 & 2534 \\
\hline Information Science Library Science & & 317 & 317 \\
\hline Mathematics & 1565 & & 1565 \\
\hline Medicine & 861 & & 861 \\
\hline
\end{tabular}




\begin{tabular}{|l|r|r|r|}
\hline Áreas & Scopus & WoS & \multicolumn{1}{c|}{ Total } \\
\hline Operations Research Management Science & & 290 & 290 \\
\hline Science Technology Other Topics & & 761 & 761 \\
\hline Social Sciences & 4402 & & 4402 \\
\hline Social Sciences Other Topics & & 301 & 301 \\
\hline Telecommunications & & 386 & 386 \\
\hline Total & $\mathbf{3 5 6 9 2}$ & $\mathbf{9 3 9 1}$ & $\mathbf{4 5 0 8 3}$ \\
\hline
\end{tabular}

Fonte: Elaboração própria.

Um artigo está vinculado a mais do que uma área, razão pela qual o total é superior ao número de publicações.

\subsubsection{Palavras-chave pesquisadas}

$\mathrm{Na}$ Figura 2 é possível observar as palavras-chave utilizadas pelos autores nas publicaçóes selecionadas.

Figura 2 - Palavras-chave pesquisadas

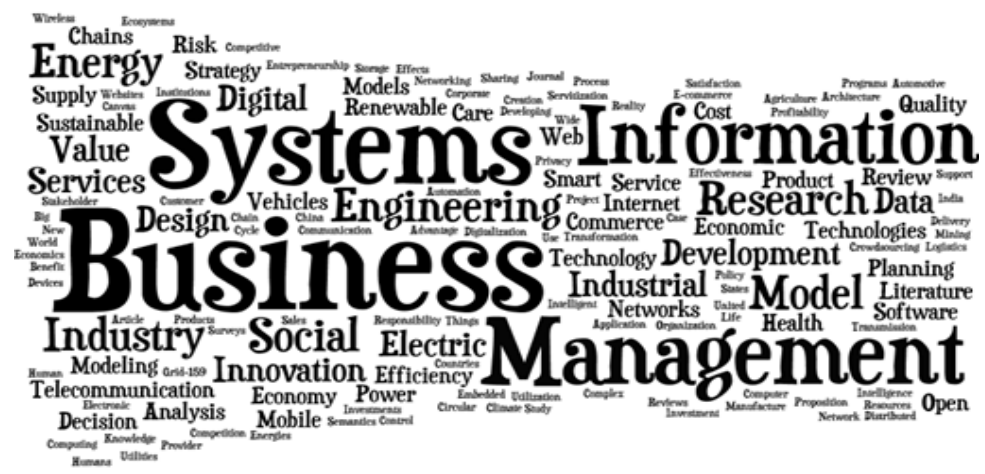

Fonte: Elaboração própria.

A figura 3 contém as 10 palavras-chave comumente verificadas nas publicaçóes da pesquisada.

Figura 3 - Frequência de palavras-chave

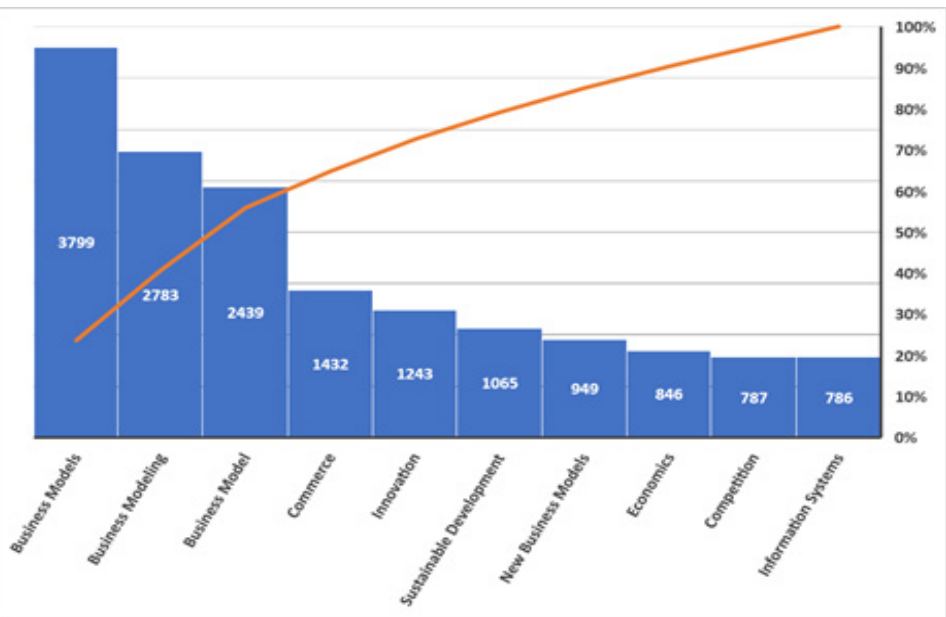

Fonte: Elaboração própria. 
Na sequência, a palavra que mais se repetiu foi "Business Models".

\subsection{Artigos mais citados no periodo em estudo}

No Quadro 9, são apresentados os 20 (vinte) artigos mais citados sobre Business Models, em cada base de dados WoS e Scopus, no período (2010 a 2019).

Destacou-se o artigo Business Models, Business Strategy and Innovation, de Teece, David J. com 4375 citaçóes, sendo 1882 na WoS e 2493 na Scopus, publicado no Long Range Planning, ano de 2010. Ainda sobre essa visão o autor com o artigo mais citado náo se encontra na lista dos autores que mais publicaram sobre o tema. Verificou-se que os artigos mais citados eram de língua inglesa, comprovando o impacto acadêmico das publicações neste idioma.

Quadro 9-Relação das 20 publicações mais citadas na WoS e Scopus

\begin{tabular}{|c|c|c|c|c|c|}
\hline WoS & Scopus & Titulo & Autor & Journal & Ano \\
\hline 1882 & 2493 & $\begin{array}{l}\text { Business Models, Business } \\
\text { Strategy and Innovation }\end{array}$ & Teece, D. J. & $\begin{array}{l}\text { Long Range Planning } \\
43(2-3), 172-194\end{array}$ & 2010 \\
\hline 1261 & 1586 & $\begin{array}{l}\text { The Business Model: Recent } \\
\text { Developments and Future } \\
\text { Research }\end{array}$ & $\begin{array}{l}\text { Zott, C.; Amit, R.; } \\
\text { Massa, L. }\end{array}$ & $\begin{array}{c}\text { Journal of Management } \\
37(4), 1019-1042\end{array}$ & 2011 \\
\hline 1065 & 1275 & $\begin{array}{l}\text { Business Model Innovation: } \\
\text { Opportunities and Barriers }\end{array}$ & Chesbrough, H. & $\begin{array}{c}\text { Long Range Planning } \\
43(2-3), 354-363\end{array}$ & 2010 \\
\hline 828 & 1098 & $\begin{array}{l}\text { From cloud computing to cloud } \\
\text { manufacturing }\end{array}$ & $\mathrm{Xu}, \mathrm{X}$. & $\begin{array}{c}\text { Robotics and Computer- } \\
\text { Integrated Manufacturing } \\
28(1), 75-86\end{array}$ & 2012 \\
\hline 772 & 867 & $\begin{array}{l}\text { A literature and practice review } \\
\text { to develop sustainable business } \\
\text { model archetypes }\end{array}$ & Bocken, et al. & $\begin{array}{l}\text { Journal of Cleaner } \\
\text { Production } \\
65,42-56\end{array}$ & 2014 \\
\hline 748 & 1102 & $\begin{array}{l}\text { Blockchains and Smart } \\
\text { Contracts for the Internet of } \\
\text { Things }\end{array}$ & $\begin{array}{l}\text { Christidis, K.; } \\
\text { Devetsikiotis, M. }\end{array}$ & $\begin{array}{l}\text { IEEE Access } \\
\text { 4, 2292-2303 }\end{array}$ & 2016 \\
\hline 649 & 804 & $\begin{array}{l}\text { From Strategy to Business } \\
\text { Models and onto Tactics }\end{array}$ & $\begin{array}{l}\text { Casadesus- } \\
\text { Masanell, R.; Enric } \\
\text { Ricart, J. }\end{array}$ & $\begin{array}{l}\text { Long Range Planning } \\
43(2-3), 195-215\end{array}$ & 2010 \\
\hline 626 & 669 & $\begin{array}{l}\text { Business models for sustainable } \\
\text { innovation: state-of-the-art and } \\
\text { steps towards a research agenda }\end{array}$ & $\begin{array}{l}\text { Boons, F.; Luedeke- } \\
\text { Freund, F. }\end{array}$ & $\begin{array}{c}\text { Journal of Cleaner } \\
\text { Production } \\
45,9-19 \\
\end{array}$ & 2013 \\
\hline 580 & 750 & The future of open innovation & $\begin{array}{l}\text { Gassmann, O.; } \\
\text { Enkel, E.; } \\
\text { Chesbrough, H. }\end{array}$ & $\begin{array}{l}\text { R \& D Management } \\
40(3), 213-221\end{array}$ & 2010 \\
\hline 515 & 685 & $\begin{array}{l}\text { Leveraging External Sources } \\
\text { of Innovation: A Review of } \\
\text { Research on Open Innovation }\end{array}$ & $\begin{array}{l}\text { West, J.; Bogers, } \\
\text { M. }\end{array}$ & $\begin{array}{c}\text { Journal of Product } \\
\text { Innovation Management } \\
31(4), 814-831 \\
\end{array}$ & 2014 \\
\hline 478 & & $\begin{array}{l}\text { The Impact of New Media on } \\
\text { Customer Relationships }\end{array}$ & $\begin{array}{l}\text { Hennig-Thurau, } \\
\text { T.;Malthouse, E.; } \\
\text { Friege, C. }\end{array}$ & $\begin{array}{c}\text { Journal of Service Research } \\
13(3), 311-330\end{array}$ & 2010 \\
\hline
\end{tabular}




\begin{tabular}{|c|c|c|c|c|c|}
\hline WoS & Scopus & Titulo & Autor & Journal & Ano \\
\hline 464 & & $\begin{array}{l}\text { Industrial Product-Service } \\
\text { Systems-IPS2 }\end{array}$ & $\begin{array}{l}\text { Meier, H.; Roy, R.; } \\
\text { Seliger, G. }\end{array}$ & $\begin{array}{c}\text { CIRP Annals- } \\
\text { Manufacturing Technology } \\
\text { 59(2),607-627 }\end{array}$ & 2010 \\
\hline 455 & & $\begin{array}{l}\text { From Multi-Channel Retailing } \\
\text { to Omni-Channel Retailing } \\
\text { Introduction to the Special Issue } \\
\text { on Multi-Channel Retailing }\end{array}$ & $\begin{array}{l}\text { Verhoef, P. C., } \\
\text { Kanna, P. K.; } \\
\text { Inman, J. J. }\end{array}$ & $\begin{array}{l}\text { Journal of Retailing } \\
91(2), 174-181\end{array}$ & 2015 \\
\hline \multirow[t]{10}{*}{449} & & $\begin{array}{l}\text { Conceptualizing the circular } \\
\text { economy: An analysis of } 114 \\
\text { definitions }\end{array}$ & $\begin{array}{l}\text { Kirchherr, J.; } \\
\text { Reike, D.; } \\
\text { Hekkert, M. }\end{array}$ & $\begin{array}{c}\text { Resources Conservation and } \\
\text { Recycling } \\
127,221-232\end{array}$ & 2017 \\
\hline & 1153 & $\begin{array}{l}\text { The Culture of Connectivity: A } \\
\text { Critical History of Social Media }\end{array}$ & Van-Dijck, J. & $1-240$ & 2013 \\
\hline & 1020 & $\begin{array}{l}\text { Business model design: An } \\
\text { activity system perspective }\end{array}$ & Zott, C., Amit, R. & $\begin{array}{l}\text { Long Range Planning } \\
43(2-3), 216-226\end{array}$ & 2010 \\
\hline & 993 & $\begin{array}{l}\text { Spreadable media: Creating } \\
\text { value and meaning in a } \\
\text { networked culture }\end{array}$ & $\begin{array}{l}\text { Jenkins, H.; Ford, } \\
\text { S.; Green, J. }\end{array}$ & pp. 1-352 & 2013 \\
\hline & 976 & $\begin{array}{l}\text { Reinventing your business } \\
\text { model }\end{array}$ & $\begin{array}{l}\text { Johnson, M. W.; } \\
\text { Christensen, C. } \\
\text { M.; Kagermann, } \\
\text { H. }\end{array}$ & $\begin{array}{l}\text { Harvard Business Review } \\
\quad 86 \text { (12), 50-59 }\end{array}$ & 2008 \\
\hline & 928 & $\begin{array}{l}\text { Open } \mathrm{R} \& \mathrm{D} \text { and open } \\
\text { innovation: Exploring the } \\
\text { phenomenon }\end{array}$ & $\begin{array}{l}\text { Enkel, E.; } \\
\text { Gassmann, O.; } \\
\text { Chesbrough, H. }\end{array}$ & $\begin{array}{l}\text { R\&D Management } \\
39(4), 311-316\end{array}$ & 2009 \\
\hline & 924 & $\begin{array}{l}\text { Sustainable supply chains: An } \\
\text { introduction }\end{array}$ & $\begin{array}{l}\text { Linton, J. D., } \\
\text { Klassen, R.; } \\
\text { Jayaraman, V. }\end{array}$ & $\begin{array}{c}\text { Journal of Operations } \\
\text { Management } \\
25(6), 1075-1082\end{array}$ & 2007 \\
\hline & 746 & $\begin{array}{l}\text { The Circular Economy - A new } \\
\text { sustainability paradigm? }\end{array}$ & Geissdoerfer, et al. & $\begin{array}{c}\text { Journal of Cleaner } \\
\text { Production, 143, 757-768. }\end{array}$ & 2017 \\
\hline & 683 & $\begin{array}{l}\text { The fit between product } \\
\text { market strategy and business } \\
\text { model: Implications for firm } \\
\text { performance }\end{array}$ & Zott, C., Amit, R. & $\begin{array}{l}\text { Strategic Management } \\
\text { Journal, 29(1), 1-26 }\end{array}$ & 2008 \\
\hline & 604 & $\begin{array}{l}\text { Business model evolution: In } \\
\text { search of dynamic consistency }\end{array}$ & $\begin{array}{l}\text { Demil, B., Lecocq, } \\
\text { X. }\end{array}$ & $\begin{array}{l}\text { Long Range Planning, } \\
43(2-3), 227-246\end{array}$ & 2010 \\
\hline
\end{tabular}

Fonte: Elaboração própria

Comparando o Quadro 9 com o Quadro 2, foi possível constatar que os autores Bocken, N.M.P., Evans, S., Gassmann, são três dos dez que mais publicaram sobre o tema no período 2010-2019, encontram-se na listagem dos autores das vinte publicaçóes mais citadas.

\section{Considerações finais}

Este estudo teve como objetivo verificar quais as características da produção científica internacional sobre o tema Modelos de Negócio. Para a realização do presente estudo efetuou-se pesquisa bibliométrica utilizando as bases de dados Web of Science e a Scopus, tendo-se obtido uma amostra final para análise de 29092 artigos. 
Os interesses e focos de investigação sobre o tema Business Models estão em crescimento para o conhecimento científico. Com isso, foi possível mapear e analisar o cenário de produção científica relacionada com o tema Business Models.

Em virtude de os artigos estarem relacionados a mais de uma área do conhecimento, considerase o tema interdisciplinar. Verificou-se que o veículo de divulgação que apresentou o maior número de publicaçóes (488) foi o Journal of Cleaner Production, seguido do Sustainability, com 422 trabalhos.

Estados Unidos é o país no qual as publicaçóes mais se concentram. Seguem outros países com menor número, tais como, Alemanha, China e Reino Unido. Observou-se multiplicidade e diversidade quanto à autoria dos trabalhos, relativamente aos autores que mais publicaram nesse tema.-

As instituições que mais se destacaram no que se refere às publicações relacionadas com Business Models foram: Aalto University (Espoo - Finlândia), University of Cambridge (Cambridge - Reino Unido), Delft University of Technology (Delft, Países Baixos), Universitat St. Gallen (St. Gallen, Suíça).

Com relação à bibliometria, é possível concluir que, no presente estudo, as Leis de Lotka ou Lei do Quadrado Inverso, Bradford ou Lei da Dispersão e de Zipf ou Lei do Mínimo Esforço, não foram confirmadas, pois grande parte da literatura científica produzida não advém de um pequeno número de autores, o que leva a interpretar que há dispersão nesse sentido; quanto à análise das publicaçóes, observouse uma grande variedade dos jornais onde os trabalhos foram publicados, mas sem haver concentraçáo de pesquisas sobre o tema em questão. A Lei de Zipf também não se pode confirmar, pois muitas palavraschave são utilizadas para referenciar as investigaçôes verificadas.

A contribuição desse estudo para as pesquisas em Gestão deve-se aos indicadores resultantes quanto às instituiçôes de pesquisa e aos periódicos que mais se destacam na produção do conhecimento sobre o tema a nível internacional.

\section{Limitações e futuras linhas de investigação}

Cabe ressaltar que embora o presente estudo tenha feito algumas contribuiçóes para a ciência, apresentou também algumas limitaçóes no que diz respeito às pesquisas efetuadas. A principal limitaçáo refere-se que a pesquisa foi realizada com artigos encontrados na base Web of Science e na Scopus. Outra limitação está no período analisado.

Sugere-se o aprimoramento da pesquisa no futuro, para obter resultados mais precisos, abrangendo, por exemplo, outras importantes bases de dados científicos, o que contribuiria para reunir mais informaçóes e alcançar uma melhor compreensão do tema. Embora tenhamos identificado os principais pontos críticos da pesquisa e sua evolução, ainda são necessárias informaçôes mais aprofundadas sobre as características da produção científica internacional sobre Modelos de Negócio, ainda são necessárias informações mais aprofundadas sobre metodologias, fundamentação teórica e os principais resultados de cada trabalho.

Nenhum investigador português ou brasileiro consta da listagem dos autores que mais publicaram sobre o tema, o que reforça a ideia de que este tema precisa ser mais estudado nos países supracitados. 


\section{Referências}

Abraham, S.W. (2013). Business model innovation replace strategic analysis? Strategy \& leadership. Emerald Group Publishing Limited 41(2), 31-38.

Araújo, C.A. (2006). Bibliometria: evolução histórica e questôes atuais. Em Questão. Enero-Junio, 12(1), 11-32.

Baden-Fuller, C., \& Morgan, M.S. (2010). Business Models as Models. Long Range Planning, Elsevier Ltd. 43(2-3), 156-171.

Bocken, N. M. P., Short, P. R., \& Evans, S. (2014). A literature and practice review to develop sustainable business model archetypes. Journal of Cleaner Production, 65: $42-56$.

Boons, F., \& Lüdeke-Freund, F. (2013). Business models for sustainable innovation: State-of the-Art and Steps Towards a Research Agenda. Journal of Cleaner Production. 45, 9-19.

Bufrem, L., \& Prates, Y. (2005). O saber científico registrado e as práticas de mensuração da informação. Ci. Inf, Brasília, 34(2), 9-25.

Campos, P. (2010). Novos modelos de negócio na indústria fonográfica: um estudo exploratórios sobre as gravadoras no Brasil. 2010. Dissertaçáo (Mestrado em Engenharia da Produção) - Universidade Federal do Rio de Janeiro, Rio de Janeiro.

Camps, D; Samar, M.E.; Ávila, R.E., \& Recuero Y. (2006). Estudio bibliométrico de un volumen de la revista Archivos de Medicina. Arch Med.; 2(3).

Casadesus-Masanell, R., \& Ricart, J. E. (2007). Competing through business models. Harvard Business School, Module Note 708-452.

Centobelli, P., Cerchione, R., Chiaroni, D., Del Vecchio, P., \& Urbinati, A., 2020. Designing business models in circular economy: a systematic literature review and research agenda. Bus. Strat. Env. 29 (4), $1734-1749$.

Chesbrough, H. (2010) Business model innovations: Opportunities and barriers. Long Range Planning, 43(2-3): 354-363.

Chesbrough, H., \& Rosenbloom, R.S. (2002). The role of the business model in capturing value from innovation: evidence from Xerox Corporation's technology spin囚off companies. Industrial and Corporate Change, 11(3), 529-555.

Christidis, K., \& Devetsikiotis, M. (2016) Blockchains and Smart Contracts for the Internet of Things. IEEE Access, 4: 2292-2303.

Demil, B., Lecocq, X. (2010). Business model evolution: In search of dynamic consistency. Long Range Planning, 43 (2-3): 227-246. 
Dorf, B., \& Blank, S. (2012). The Startup Owner's Manual - the Step-by-Step Guide for Building a Great Company. K\&Ranch, Inc. Publishers. Pescadero, California, USA -. 571p.

Drucker, P. (1973). A teoria do negócio. USA: Harvard Business Review. Reimpress 2019 Actual Editora.

Edgeman R., \& Eskildsen J. (2014). Modeling and assessing sustainable enterprise excellence. Business Strategy and the Environment 23(3): 173-187.

Enkel, E., Gassmann, O., \& Chesbrough, H. (2009). Open R\&D and open innovation: Exploring the phenomenon. R\&D Management, 39(4): 311-316.

Evans, S., Vladimirova, D., Holgado, M., Van Fossen, K., Yang, M., Silva, E.A., \& Barlow, C.Y. (2017). Business model innovation for sustainability: towards a unified perspective for creation of sustainable business models. Bus. Strategy Environ. 26(5), 597-608.

Gassman, O., Enkel, E., \& Chesbrough, H. (2010). The future of open innovation. $R \& D$ Management, 40(3), 213-221.

Geissdoerfer, M.; Savaget, P., Bocken, N. M. P., \& Hultink, E. J. (2017). The Circular Economy - A new sustainability paradigm? Journal of Cleaner Production, 143: 757-768.

Hamel, G. (2000). Leading the revolution. Boston: Harvard Business School.

Hennig-Thurau, T., Malthouse, E., \& Friege, C. (2010). The Impact of New Media on Customer Relationships. Journal of Service Research, 13(3): 311-330.

Jenkins, H., Ford, S., \& Green, J. (2013). Spreadable media: Creating value and meaning in a networked culture. USA: New York University Press.

Johnson, M. W., Christensen, C. M., \& Kagermann, H. (2008). Reinventing your business model. Harvard Business Review, 86 (12): 50-59.

Kirchherr, J., Reike, D., \& Hekkert, M. (2017). Conceptualizing the circular economy: An analysis of 114 definitions, Resources Conservation and Recycling, 127: 221-232.

Lambert, S.C., (2006), “Do We Need a 'Real' Taxonomy of e-Business Models?” School of Commerce Research Paper Series, 06-06.

Lind, J. (2004). A Business model definition: validating opportunities opened by technological change. International Journal of Eletronic Commerce.

Linder, M., \& Williander, M. (2017). Circular Business Model Innovation: Inherent Uncertainties. Business Strategy and the Environment, 26(2), 182-196.

Linton, J. D., Klassen, R., \& Jayaraman, V. Sustainable supply chains: An introduction. Journal of Operations Management, 25(6): 1075-1082. 
Machado, R. D.N. (2007). Análise cientométrica dos estudos bibliométricos publicados em periódicos da área de biblioteconomia e ciência da informação. (1990-2005). Perspectivas em Ciência da Informação, $12(3), 2-20$.

Magretta, J. (2002). Why business models matter. Harvard Business Review, 80(5), 86-92.

Meier, H., Roy, R., \& Seliger, G. (2010). Industrial Product-Service Systems-IPS2. CIRP AnnalsManufacturing Technology, 59(2): 607-627.

Orofino, M.A.R. (2011). Técnicas de criação do conhecimento no desenvolvimento de modelos de negócio. Dissertação (Mestrado). Centro Tecnológico, Programa de Pós-Graduaçáo em Engenharia e Gestão do Conhecimento., Universidade Federal de Santa Catarina.

Osterwalder, A. (2004). The business model ontology - a proposition in a design science approach. Ph.D. (Thesis). University of Lausanne, Ecole des Hautes 74 Etudes Commerciales HEC, Lausanne, Switzerland.

Osterwalder, A., \& Pigneur, Y. (2009). Business Model Generation. OSF.

Osterwalder, A., \& Pigneur, Y. (2011). Business Model Generation - inovação em modelos de negócios: um manual para visionários, inovadores e revolucionários. Alta Books.

Osterwalder, A., \& Pigneur, Y. (2013). Designing business models and similar strategic objects: the contribution of IS. Journal of the Association for Information Systems, 14(5), 237-244.

Rousseau R. (2001). Indicadores bibliométricos y econométricos en la evaluación de instituciones científicas. ACIMED. 9, 23-9.

Rousseau, B., \& Rousseau, R. (2000). Percolation as a model for informetric distributions: fragment size distribution characterized by Bradford curves". Scientometrics, [S.1.], 47, 195-206.

Schaltegger, S.; Hansen, E. G., \& Lüdeke-Freund, F. (2016). Business Models for Sustainability: Origins, Present Research, and Future Avenues. Organization \& Environment, 29(1), 3-10.

Schneider, S., \& Spieth, P. (2013). Business Model Innovation: Towards an Integrated Future Research Agenda. International Journal of Innovation Management, 17(1), 1340001 (34 pages).

Teece, D. J. (2010). Business models, business strategy and innovation. Long Range Planning, 43(n. 2-3), 172-194.

Van-Dijck, J. (2013). The Culture of Connectivity: A Critical History of Social Media. USA: Oxford University Press.

Verhoef, P.C., Kannan, P. K., \& Inman, J. J. (2015). From Multi-Channel Retailing to Omni-Channel Retailing Introduction to the Special Issue on Multi-Channel Retailing. Journal of Retailing, 91(2): 174181. 
West, J.; Bogers, M. (2014). Leveraging External Sources of Innovation: A Review of Research on Open Innovation. Journal of Product Innovation Management, 31(4): 814-831.

$\mathrm{Xu}, \mathrm{X}$. (2012). From cloud computing to cloud manufacturing. Robotics and Computer-integrated Manufacturing, 28(1), 75-86.

Yang M, Evans S, Vladimirova D, \& Rana P. (2017). Value uncaptured perspective for sustainable business model innovation. Journal of Cleaner Production, 140, 1794-1804.

Zott, C.; Amit, R. (2010). Business model design: An activity System Perspective. Long Range Planning, 43(2-3), 216-226.

Zott, C., Amit, R., \& Massa, L. (2011). The business model: theoretical roots, recent developments, and future research. Journal of Management. 37(4), 1019-1042. 